\title{
Involvement of IL-10 and granulocyte colony-stimulating factor in the fate of monocytes controlled by galectin-1
}

\author{
DA-EN CHENG $^{1 *}$, WEI-AN CHANG ${ }^{2,3^{*}}$, JEN-YU HUNG $^{3,4}$, MING-SHYAN HUANG $^{3,4}$ and PO-LIN KUO ${ }^{2,5}$ \\ ${ }^{1}$ Graduate Institute of Medicine; ${ }^{2}$ Institute of Clinical Medicine, College of Medicine, Kaohsiung Medical University, \\ Kaohsiung 807; ${ }^{3}$ Division of Pulmonary and Critical Care Medicine, Kaohsiung Medical University Hospital, \\ Kaohsiung 807; ${ }^{4}$ School of Medicine, College of Medicine, Kaohsiung Medical University, Kaohsiung 807; \\ ${ }^{5}$ Department of Medical Research, Kaohsiung Medical University Hospital, Kaohsiung 807, Taiwan, R.O.C.
}

Received November 30, 2013; Accepted June 5, 2014

DOI: $10.3892 / \mathrm{mmr} .2014 .2573$

\begin{abstract}
The process of differentiation from monocytes to dendritic cells is critical in immune modulation. Monocyte apoptosis is a key regulator in balancing the immune response. Galectin-1 has been reported to induce tolerogenic dendritic cells by the autocrine interleukin (IL)-10 in monocytes. However, IL-10 has been found to induce apoptosis in IL-4/granulocyte macrophage colony-stimulating factor (CSF) stimulating and non-stimulating monocytes, whereas galectin-1 has not. After analyzing the factors secreted by galectin-1-activated CD14 monocytes isolated from the peripheral blood, the present study revealed that galectin-1 upregulates IL-10 and granulocyte (G)-CSF expression. Furthermore, G-CSF inhibited IL-10-induced apoptosis, implying that galectin-1 may enhance the immune-modulating functions of G-CSF by inducing tolerogenic dendritic cells and maintaining their survival. Therefore, G-CSF may be further applied in immune therapy, particularly in the IL-10-presenting microenvironment.
\end{abstract}

\section{Introduction}

Dendritic cells (DCs) are specific antigen-presenting cells critical for the induction of adaptive immunity and tolerance by interacting with T cells (1). DC differentiation from monocytes is a key step in infections and numerous other conditions. DC turnover is similarly important for maintaining the steady state of the immune system. Circulating monocytes usually undergo spontaneous apoptosis within days (2); however,

Correspondence to: Professor Po-Lin Kuo, Institute of Clinical Medicine, College of Medicine, Kaohsiung Medical University, 100 Shih-Chuan 1st Road, Kaohsiung 807, Taiwan, R.O.C.

E-mail:kuopolin@seed.net.tw

${ }^{*}$ Contributed equally

Key words: galectin-1, monocyte, IL-10, granulocyte colony-stimulating factor, apoptosis the life span of monocytes is extended to weeks following differentiation into DCs, induced by treatment with interleukin (IL)-4/granulocyte macrophage colony-stimulating factor (GM-CSF).

Previous studies have observed that T-helper (Th)1 cytokines, including IL-2 and IL-12, inhibit myeloid cell apoptosis, whereas Th 2 cytokines, such as IL-4 and IL-10, enhance apoptosis in these cells $(3,4)$. IL-10-induced myeloid cell apoptosis is mediated through the caspase-dependent signaling pathway, which is blocked by caspase- 3 inhibitors and pan-caspase inhibitors (2). Galectin-1 (Gal-1) exhibits the ability to induce IL-10 expression in T cells $(5,6)$ and in DCs $(7,8)$, but does not induce apoptosis in monocytes $(9,10)$.

Granulocyte colony-stimulating factor (G-CSF, also termed CSF3) was identified in an attempt to define the normal regulators present in cell supernatants that induced terminal differentiation of the WEHI-3B $\mathrm{D}^{+}$murine myeloid leukemia cell line (11). Recently, Romero-Weaver et al reported the ability of G-CSF to promote the proliferation of bone marrow stem cells and inhibit granulocyte apoptosis (12). G-CSF also improved the recovery from spinal cord injury in mice (13) and improved memory and neuro-behavior in an amyloid- $\beta$-induced experimental model of Alzheimer's disease (14). However, the direct effects of G-CSF on differentiating monocytes have not been discussed. In present study, the role of G-CSF in galectin-1-treated monocytes was examined, particularly its role in preventing cell apoptosis.

\section{Materials and methods}

Materials. Gal-1 and G-CSF were purchased from ProsPec-Tany TechnoGene, Ltd. (Ness-Ziona, Israel). Human recombinant IL-10 was purchased from R\&D Systems (Minneapolis, MN, USA). Human recombinant GM-CSF and IL-4 were purchased from Millipore Corp. (Billerica, MA, USA).

Isolation and culture of human monocytes. Human CD14 ${ }^{+}$ monocytes were isolated from the peripheral blood mononuclear cells (PBMCs) of healthy donors without any known cancers or immunological disease. Briefly, PBMCs were collected from interphase subsequent to Ficoll paque plus separation (GE 
A

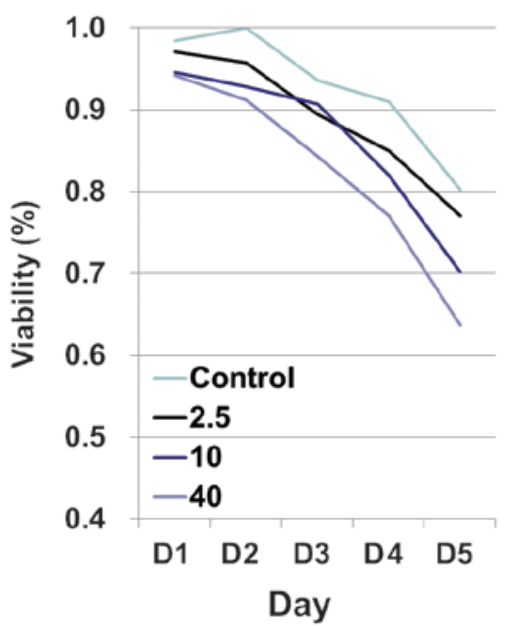

C

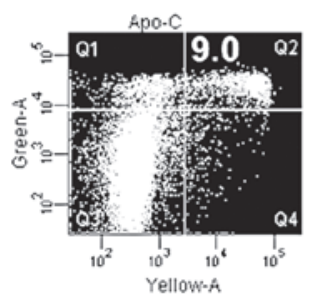

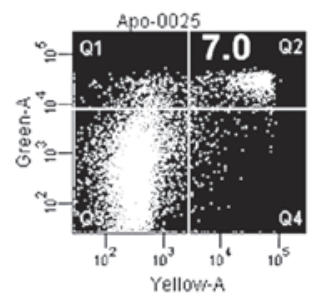

B
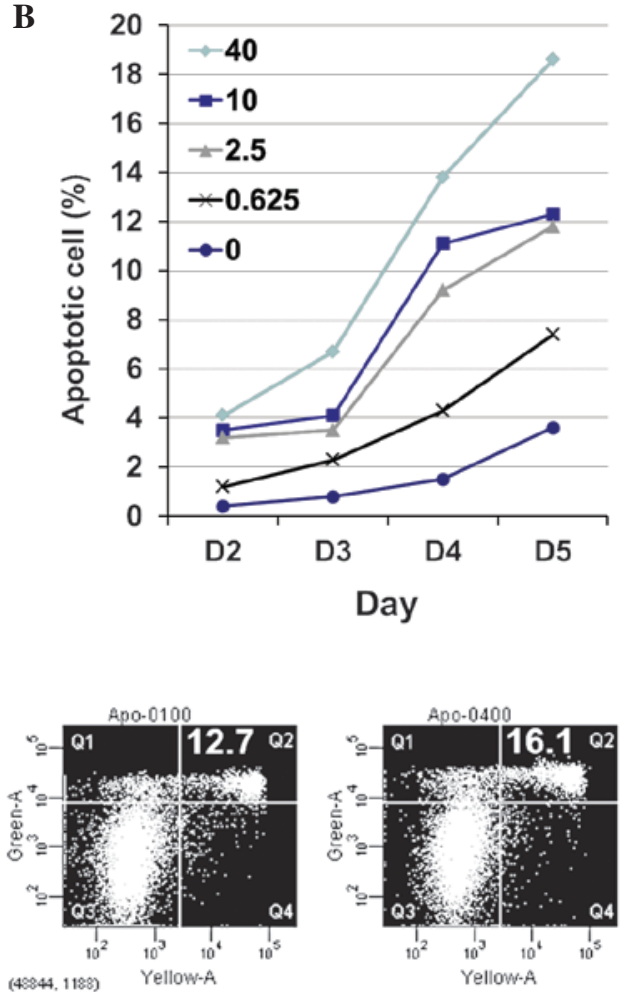

Figure 1. IL-10 induces apoptosis in IL-4/GM-CSF-stimulated monocytes. (A) Different concentrations of IL-10 (2.5, 10 and 40 ng/ml) were added to the culture media and cell viability was determined by trypan blue exclusion assay at various time-points. (B and C) Stimulated monocytes from different treatments were placed into a 96-well plate, stained with fluorescein isothiocyanate-conjugated Annexin V and propidium iodide and analyzed by flow cytometry. The results of single experiments are shown and all the results are representative of at least three independent experiments.. GM-CSF, granulocyte macrophage colony-stimulating factor; IL, interleukin.

Healthcare Bio-Sciences, Little Chalfont, UK) and washed twice in phosphate-buffered saline (PBS). CD14 ${ }^{+}$monocytes were isolated using the MACS ${ }^{\circledR}$ system (MACS MicroBeads; Miltenyi Biotec Ltd, Bergisch Gladbach, Germany) following the manufacturer's instructions and cultured in RPMI-1640 containing $10 \%$ fetal bovine serum (Invitrogen Life Sciences, Carlsbad, CA, USA) for five days in the presence of $20 \mathrm{ng} / \mathrm{ml}$ IL-4/GM-CSF with or without $1 \mu \mathrm{g} / \mathrm{ml} \mathrm{Gal}-1,10 \mathrm{ng} / \mathrm{ml} \mathrm{G-CSF}$ and IL-10 as indicated. Monocyte viability was determined by trypan blue exclusion staining.

The Institutional Review Board of Kaohsiung Medical University Hospital (Kaohsiung, Taiwan) approved the study. All patients provided informed consent in accordance with the Declaration of Helsinki.

Flow cytometry and detection of Annexin $V$ staining and CD14 expression. Two-color flow cytometry was performed by FACSarray $^{\text {TM }}$ (BD Biosciences, Franklin Lakes, NJ, USA) using the Annexin V-fluorescein isothiocyanate (FITC) Apoptosis Detection kit I (BD Biosciences) according to the manufacturer's instructions. Briefly, the treated cells were centrifuged at $200 \mathrm{x}$ g for $5 \mathrm{~min}$ and washed twice with cold PBS. The cells were resuspended in $100 \mu 1 \mathrm{XX}$ binding buffer, and $5 \mu \mathrm{l}$ Annexin V-FITC and $5 \mu \mathrm{l}$ propidium iodide (PI) were added. The cells were gently vortexed and incubated for $15 \mathrm{~min}$ at room temperature in the dark. Subsequently, the cells were centrifuged at $200 \mathrm{x} g$ for $5 \mathrm{~min}$, washed twice with $1 \mathrm{X}$ binding buffer and resuspended in $100 \mu \mathrm{l} 1 \mathrm{X}$ binding buffer. The samples were analyzed using a FACSarray ${ }^{\mathrm{TM}}$ flow cytometer.

Measurement of secreted factors. The cultured supernatants from monocytes were collected following centrifugation. The samples were analyzed for IL-10 and G-CSF by multiple cytokine analyses using the cytometric bead array (CBA; BD Biosciences). The CBA technique is based on two bead populations with distinct fluorescence intensities that are coated with capture antibodies specific for each cytokine. The fluorescent dye had a maximal emission wavelength of $\sim 650 \mathrm{~nm}$ (FL-3), which was detectable by flow cytometry. The cytokine capture beads were mixed with the phycoerythrin-conjugated detection antibodies and then incubated with recombinant standards or test samples to form sandwich complexes. Following the acquisition of sample data on the FACSarray ${ }^{\text {TM }}$ flow cytometer, the sample results were analyzed using FCAP Array ${ }^{\mathrm{TM}}$ software version 3.0 (BD Biosciences). A standard calibration curve was established for each cytokine; the maximum and minimum limits of detection for each cytokine were 1.0 and $5,000 \mathrm{pg} / \mathrm{ml}$, respectively.

Statistical analysis. Data are expressed as the mean \pm standard deviation. Statistical comparisons of the results were performed by analysis of variance and two-sided Student's t-test using Excel 2010 (Microsoft Corp., Redmond, WA, USA). $\mathrm{P}<0.05$ was considered to indicate a statistically significant difference between the means of the two groups. 
A

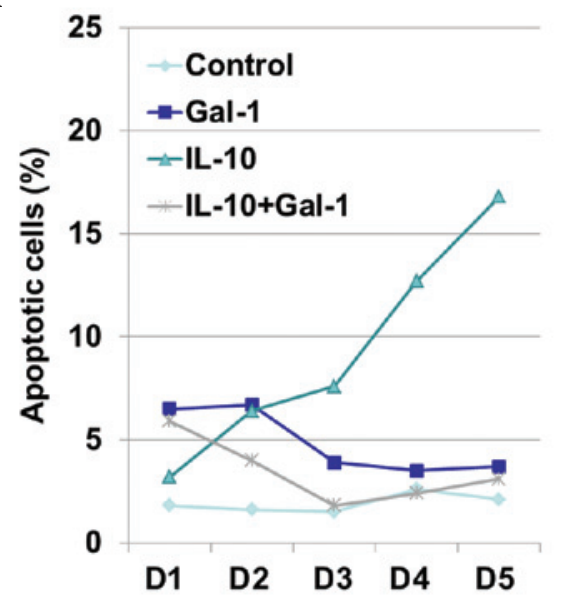

B

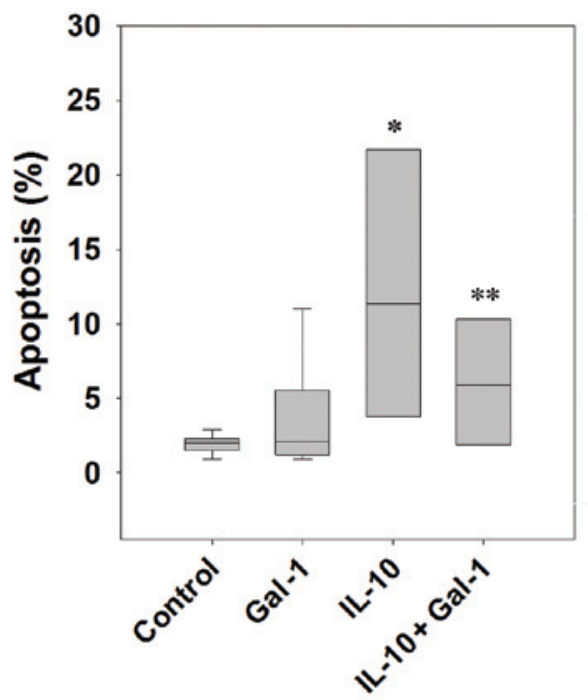

Figure 2. Gal-1 inhibits IL-10-induced apoptosis in stimulated monocytes. (A) The percentage of apoptotic cells was determined by Annexin V-propidium iodide staining at each time-point. (B) The percentage of monocyte apoptosis following different treatments on day five was determined in five healthy donors. Each value signifies the mean \pm standard deviation of five independent experiments. ${ }^{*} \mathrm{P}<0.05$ vs. control; ${ }^{* *} \mathrm{P}<0.05$ vs. IL-10 group, by Kruskal-Wallis non-parametric analysis of variance. Gal-1, galectin-1; IL, interleukin.

A

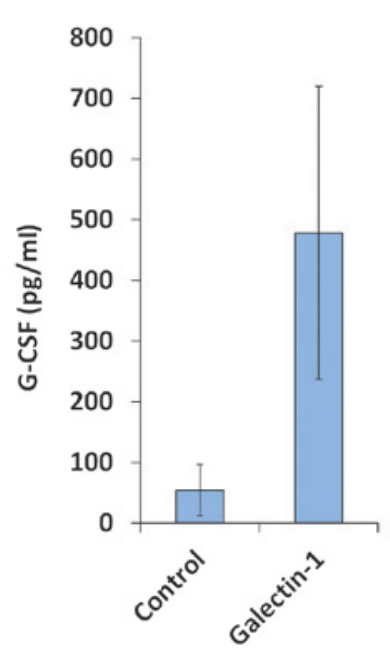

B

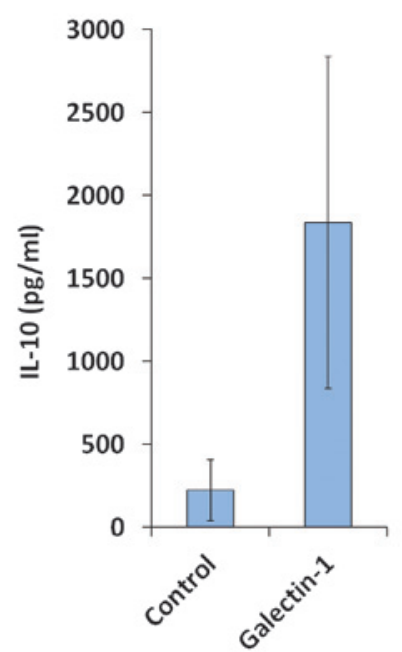

C

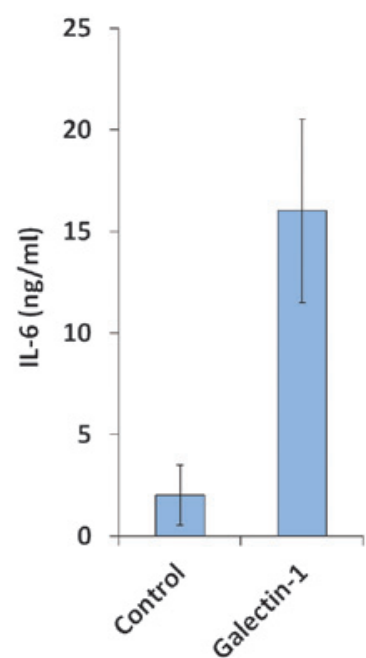

Figure 3. Galectin-1 induces IL-10 and G-CSF in stimulated monocytes. (A-C) Cytokine concentrations as determined by cytometry bead array. All results are representative of at least three independent experiments. G-CSF, granulocyte colony-stimulating factor; IL, interleukin.

\section{Results}

IL-10 induces apoptosis in monocytes. Monocytes isolated from PBMCs of healthy donors usually died after several days due to a constitutively active cell death program (15). This spontaneous cell death was reduced by $20 \%$ following stimulation with IL-4 and GM-CSF for five days (Fig. 1A). The viability of the stimulated monocytes, determined by trypan blue exclusion assay, was reduced when IL-10 was added and the proportion of trypan blue- stained cells increased following treatment with higher IL-10 concentrations (Fig. 1A). Similarly, Annexin V-PI staining revealed that the proportion of apoptotic cells was elevated with increasing IL-10 concentration and increased culture duration (Fig. 1B and C). The apoptosis induced by recombinant human IL-10 was significantly increased at concentrations $>2.5 \mathrm{ng} / \mathrm{ml}$.
Gal-1 protects monocytes from IL-10-induced apoptosis. The percentage of apoptotic cells was determined by Annexin V-propidium iodide staining of the IL-4/GM-CSF-stimulating monocyte culture media with and without $1 \mu \mathrm{g} / \mathrm{ml} \mathrm{Gal}-1$ and/or $10 \mathrm{ng} / \mathrm{ml}$ IL-10. Stimulated monocyte apoptosis in the IL-10-only group continuously increased over five days. The Gal-1-only group exhibited no increase in apoptosis after three days (Fig. 2A). Furthermore, IL-10+Gal-1-stimulated monocyte apoptosis was not increased after three days (Fig. 2A). The same phenomenon was observed in monocytes isolated from five donors, although the percentage of apoptotic cells varied (Fig. 2B).

Gal-1 induces IL-10 and G-CSF in stimulated monocytes. The supernatants of the Gal-1 only group were collected after five days of incubation and analyzed by the CBA system. The 
A

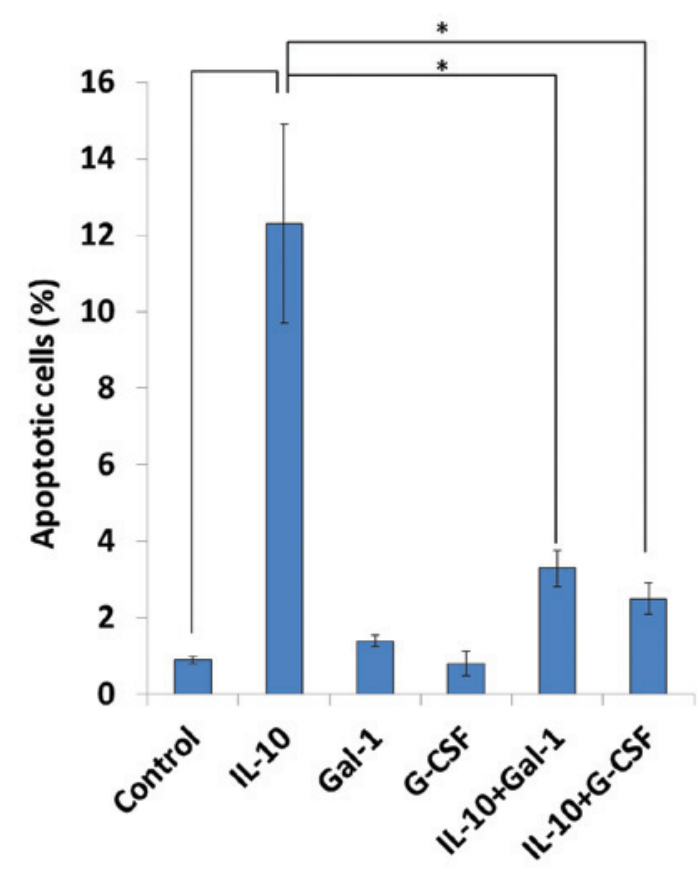

B

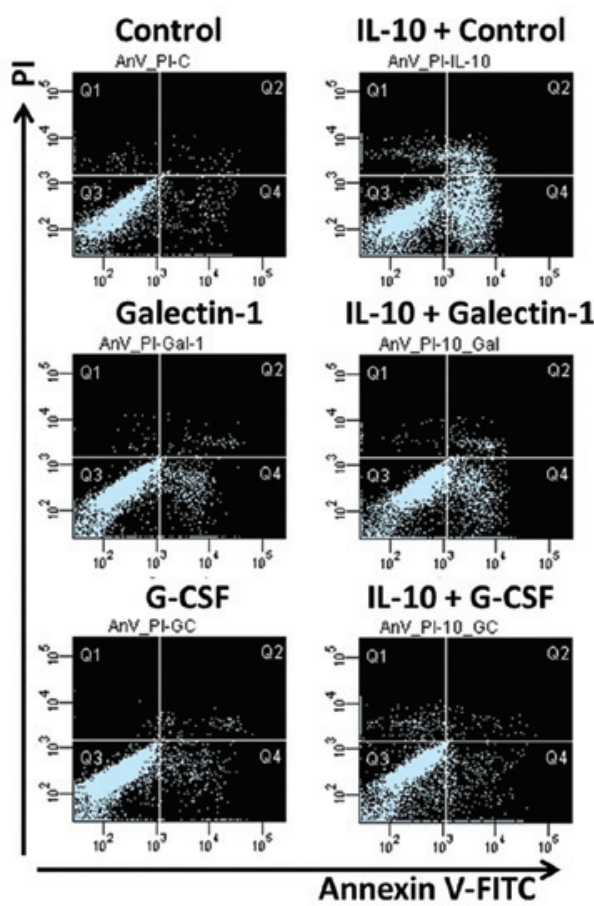

Figure 4. G-CSF inhibits IL-10-induced apoptosis in stimulated monocytes. (A-B) The percentage of apoptotic cells on day five was determined by Annexin V-PI staining and repeated in three healthy donors. * $\mathrm{P}<0.05$ vs. IL-10-only group, as detemined by one-way analysis of variance. G-CSF, granulocyte colony-stimulating factor; Gal-1, galectin-1; IL, interleukin; FITC, fluorescein isothiocyanate; PI, propidium iodide.

concentrations of $>10$ cytokines (i.e. IL-1, $-4,-6,-8,-10,-11$, $-12,-17$ and -21 , interferons (IFNs), the tumor necrosis factors (TNFs), basic fibroblast growth factor, vascular endothelial growth factor and G-CSF) were determined, with GM-CSF serving as an internal control. Gal-1 enhanced the expression levels of IL-6, IL-10 and G-CSF, but not those of the other cytokines (Fig. 3A-C).

G-CSF inhibits IL-10-induced apoptosis in monocytes. When IL-10 (10 ng/ml) was added to the IL-4/GM-CSF-stimulated monocyte culture media with and without Gal-1 $(1 \mu \mathrm{g} / \mathrm{ml})$ and G-CSF (10 ng/ml), analysis of stimulated monocyte apoptosis revealed that recombinant human G-CSF or Gal-1 significantly inhibited IL-10-induced apoptosis $(\mathrm{P}<0.05$ as compared with IL-10-only treated cells; Fig. 4A and B).

\section{Discussion}

The fate of monocytes is regulated by different signaling pathways, including those of NF- $\mathrm{B}$, Fas-Fas ligand (FasL) and the B-cell lymphoma 2 (Bcl-2) family. A previous study reported that spontaneous monocyte apoptosis was inhibited by treatment with inflammatory mediators, including TNF, lipopolysaccharide (LPS), CD40 ligand (CD154), growth factors and cytokines, including GM-CSF and IFN- $\gamma(16)$. Alone, IL-4 does not inhibit spontaneous apoptosis, and may inhibit the anti-apoptotic effects of IL-1 and LPS (3,17). However, co-treatment with GM-CSF and IL-4, according to the monocyte-derived DC protocol, inhibits the spontaneous apoptosis of monocytes (17). This implies that the signaling pathway involved in the anti-apoptotic effect mediated by GM-CSF may be different from the signaling pathway induced by IL-1 and LPS.
Receptors of pro-inflammatory mediators, including TNF receptor, IL-1R, Toll-like receptor 4 and CD14, activate the $\mathrm{NF}-\kappa \mathrm{B}$ signaling pathway and upregulate anti-apoptotic genes (18). Conversely, the GM-CSF receptor activates the Janus kinase (JAK)/signal transducer and activator of transcription (STAT)5 signaling pathway and upregulates Bcl-2 in neural progenitor cells and mouse hematopoietic precursors $(19,20)$.

Studies regarding IL-4 and IL-6 in monocytes support the hypothesis that IL-4 inhibits IL-6 production by reducing nuclear $\mathrm{NF}-\kappa \mathrm{B}$ levels $(21,22)$. However, the interaction between the IL-4 signaling pathway and STAT5 in monocytes has not been reported. Notably, in the present study, apoptosis enhanced by another Th2 cytokine, IL-10, was not inhibited by the presence of GM-CSF, suggesting a difference between IL-10-induced apoptosis and apoptosis enhanced by IL-4. Hashimoto et al (23) obtained similar results and further demonstrated that IL-10 inhibited the phosphorylation of STAT5 induced by GM-CSF. In another study, Schmidt et al (24) found that CD95 ligand-neutralizing antibody significantly inhibited IL-10-induced apoptosis. In conclusion, IL-10 may induce apoptosis by inhibiting STAT5 and by activating the Fas/FasL signaling pathway.

Galectins are a family of $15 \beta$-galactoside-binding proteins. Gal-1 is a $14.5 \mathrm{kDa}$ protein and was the first galectin family member to be described. Dimeric Gal-1 binds to glycoproteins and activates signaling pathways, including those of CD4, CD7, CD43 and CD45 (25-28). Numerous studies have demonstrated that Gal-1 induces apoptosis in T cells $(25,28-32)$ and macrophages (33), which may be involved in the regulation of immune responses. The signaling pathway involved in Gal-1-mediated T-cell 
death requires clarification, as data remain inconclusive due to variations in Gal-1 interacting proteins and concentrations (34).

A study revealed that Gal-1 regulates the T-cell immune response through upregulating IL-10 expression; Gal-1 did not induce apoptosis in myeloid lineage and Th cells, but did increase the regulatory T-cell population (35). In another model, recombinant Gal-1 enhanced IL-10 expression levels up to seven-fold, but the apoptosis induced by high dosages of IL-10 was not observed, implying that other signaling pathways activated by Gal-1 inhibit the pro-apoptotic effects of IL-10 (36). In the present study, Gal-1 enhanced IL-6 and G-CSF expression levels up to twelve- and nine-fold, respectively, but not the expression levels of pro-inflammatory cytokines (i.e. TNF, IFN and IL-12; data not shown). Mangan and Wahl (37) reported that IL-6 exerted no effect on non-stimulating apoptosis; this was also observed in later studies $(5,6)$. The present study demonstrated that IL-6 did not inhibit IL-10-induced apoptosis in IL-4/GM-CSF-stimulated monocytes. However, another hematopoietic growth factor induced by Gal-1, G-CSF, was found to reduce IL-10-induced apoptosis.

G-CSF is the predominant regulator of neutrophil production under basal conditions of hematopoiesis. G-CSF maintains neutrophil survival $(38,39)$ and regulates the survival and mobilization of cardiomyocytes and neurons (40-42). The G-CSF receptor belongs to the cytokine receptor type I superfamily, which engages the canonical JAK/STAT, Ras/Raf/mitogen-activated protein kinase and protein kinase B signaling pathways, all of which are crucial for the anti-apoptotic function of G-CSF $(43,44)$.

The present study demonstrated that G-CSF not only exerted an anti-apoptotic effect on monocytes, but also inhibited IL-10-induced apoptosis without affecting the tolerogenic function of IL-10 (data not shown). Examining the network of cytokines that regulate the fate of monocytes, this implies that Gal-1 reinforces its immune modulating effects by simultaneously upregulating IL-10 and G-CSF. Therefore, G-CSF may be further applied in immune therapy, particularly in the IL-10-presenting microenvironment.

\section{Acknowledgements}

This study was supported by grants from the National Science Council of Taiwan (no. NSC 101-2628-B-037-001-MY3) and the Kaohsiung Medical University Hospital (no. KMUH102-2M09). The authors would like to thank the Centre for Resources, Research and Development of Kaohsiung Medical University for support with the instrumentation.

\section{References}

1. Janikashvili N, Bonnotte B, Katsanis E and Larmonier N: The dendritic cell-regulatory $\mathrm{T}$ lymphocyte crosstalk contributes to tumor-induced tolerance. Clin Dev Immunol 2011: 430394, 2011

2. Fahy RJ, Doseff AI and Wewers MD: Spontaneous human monocyte apoptosis utilizes a caspase-3-dependent pathway that is blocked by endotoxin and is independent of caspase-1 J Immunol 163: 1755-1762, 1999.

3. Estaquier J and Ameisen JC: A role for T-helper type-1 and type- 2 cytokines in the regulation of human monocyte apoptosis. Blood 90: 1618-1625, 1997.
4. Ludewig B, Graf D, Gelderblom HR, Becker Y, Kroczek RA and Pauli G: Spontaneous apoptosis of dendritic cells is efficiently inhibited by TRAP (CD40-ligand) and TNF-alpha, but strongly enhanced by interleukin-10. Eur J Immunol 25: 1943-1950, 1995.

5. van der Leij J, van den Berg A, Harms G, et al: Strongly enhanced IL-10 production using stable galectin-1 homodimers. Mol Immunol 44: 506-513, 2007.

6. van der Leij J, van den Berg A, Blokzijl T, et al: Dimeric galectin-1 induces IL-10 production in T-lymphocytes: an important tool in the regulation of the immune response. J Pathol 204: 511-518, 2004.

7. Kuo PL, Hung JY, Huang SK, et al: Lung cancer-derived galectin-1 mediates dendritic cell anergy through inhibitor of DNA binding 3/IL-10 signaling pathway. J Immunol 186: 1521-1530, 2011.

8. Ilarregui JM, Croci DO, Bianco GA, et al: Tolerogenic signals delivered by dendritic cells to $\mathrm{T}$ cells through a galectin-1-driven immunoregulatory circuit involving interleukin 27 and interleukin 10. Nat Immunol 10: 981-991, 2009.

9. Stowell SR, Qian Y, Karmakar S, et al: Differential roles of galectin-1 and galectin-3 in regulating leukocyte viability and cytokine secretion. J Immunol 180: 3091-3102, 2008.

10. Barrionuevo P, Beigier-Bompadre M, Ilarregui JM, et al: A novel function for galectin-1 at the crossroad of innate and adaptive immunity: galectin-1 regulates monocyte/macrophage physiology through a nonapoptotic ERK-dependent pathway. J Immunol 178: 436-445, 2007.

11. Welte K, Platzer E, Lu L, et al: Purification and biochemical characterization of human pluripotent hematopoietic colony-stimulating factor. Proc Natl Acad Sci USA 82: 1526-1530, 1985.

12. Romero-Weaver AL, Wan XS, Diffenderfer ES, Lin L and Kennedy AR: Kinetics of neutrophils in mice exposed to radiation and/or granulocyte colony-stimulating factor treatment. Radiat Res 180: 177-188, 2013.

13. Guo Y, Zhang H, Yang J, et al: Granulocyte colony-stimulating factor improves alternative activation of microglia under microenvironment of spinal cord injury. Neuroscience 15: 238, 2013.

14. Prakash A, Medhi B and Chopra K: Granulocyte colony stimulating factor (GCSF) improves memory and neurobehavior in an amyloid- $\beta$ induced experimental model of Alzheimer's disease. Pharmacol Biochem Behav 110: 46-57, 2013.

15. Doseff AI: Apoptosis: the sculptor of development. Stem Cells Dev 13: 473-483, 2004.

16. Kiener PA, Davis PM, Starling GC, et al: Differential induction of apoptosis by Fas-Fas ligand interactions in human monocytes and macrophages. J Exp Med 185: 1511-1516, 1997.

17. Mangan DF, Robertson B and Wahl SM: IL-4 enhances programmed cell death (apoptosis) in stimulated human monocytes. J Immunol 148: 1812-1816, 1992.

18. Gaur U and Aggarwal BB: Regulation of proliferation, survival and apoptosis by members of the TNF superfamily. Biochem Pharmacol 66: 1403-1408, 2003.

19. Choi JK, Kim KH, Park H, Park SR and Choi BH: Granulocyte macrophage-colony stimulating factor shows anti-apoptotic activity in neural progenitor cells via JAK/STAT5-Bcl-2 pathway. Apoptosis 16: 127-134, 2011.

20. Feldman GM, Rosenthal LA, Liu X, et al: STAT5A-deficient mice demonstrate a defect in granulocyte-macrophage colony-stimulating factor-induced proliferation and gene expression. Blood 90: 1768-1776, 1997.

21. Donnelly RP, Crofford LJ, Freeman SL, et al: Tissue-specific regulation of IL-6 production by IL-4. Differential effects of IL-4 on nuclear factor-kappa B activity in monocytes and fibroblasts. J Immunol 151: 5603-5612, 1993.

22. Takeshita S, Gage JR, Kishimoto T, Vredevoe DL and Martínez-Maza O: Differential regulation of IL-6 gene transcription and expression by IL-4 and IL-10 in human monocytic cell lines. J Immunol 156: 2591-2598, 1996.

23. Hashimoto SI, Komuro I, Yamada M and Akagawa KS: IL-10 inhibits granulocyte-macrophage colony-stimulating factor-dependent human monocyte survival at the early stage of the culture and inhibits the generation of macrophages. J Immunol 167: 3619-3625, 2001.

24. Schmidt M, Lügering N, Pauels HG, Schulze-Osthoff K, Domschke W and Kucharzik T: IL-10 induces apoptosis in human monocytes involving the CD95 receptor/ligand pathway. Eur J Immunol 30: 1769-1777, 2000.

25. Nguyen JT, Evans DP, Galvan M, et al: CD45 modulates galectin-1-induced $\mathrm{T}$ cell death: regulation by expression of core 2 O-glycans. J Immunol 167: 5697-5707, 2001. 
26. Pang M, He J, Johnson P and Baum LG: CD45-mediated fodrin cleavage during galectin-1 $\mathrm{T}$ cell death promotes phagocytic clearance of dying cells. J Immunol 182: 7001-7008, 2009.

27. Fulcher JA, Chang MH, Wang S, et al: Galectin-1 co-clusters CD43/CD45 on dendritic cells and induces cell activation and migration through Syk and protein kinase $\mathrm{C}$ signaling. J Biol Chem 284: 26860-26870, 2009.

28. Perillo NL, Pace KE, Seilhamer JJ and Baum LG: Apoptosis of T cells mediated by galectin-1. Nature 378: 736-739, 1995.

29. Stillman BN, Hsu DK, Pang M, et al: Galectin-3 and galectin-1 bind distinct cell surface glycoprotein receptors to induce $\mathrm{T}$ cell death. J Immunol 176: 778-789, 2006.

30. Garín MI, Chu CC, Golshayan D, Cernuda-Morollón E, Wait R and Lechler RI: Galectin-1: a key effector of regulation mediated by $\mathrm{CD}^{+}{ }^{+} \mathrm{CD} 25^{+} \mathrm{T}$ cells. Blood 109: 2058-2065, 2007.

31. Pace KE, Hahn HP, Pang M, Nguyen JT and Baum LG: CD7 delivers a pro-apoptotic signal during galectin-1-induced $\mathrm{T}$ cel death. J Immunol 165: 2331-2334, 2000.

32. Perillo NL, Uittenbogaart CH, Nguyen JT and Baum LG: Galectin-1, an endogenous lectin produced by thymic epithelial cells, induces apoptosis of human thymocytes. J Exp Med 185: 1851-1858, 1997.

33. Paclik D, Werner L, Guckelberger O, Wiedenmann B and Sturm A: Galectins distinctively regulate central monocyte and macrophage function. Cell Immunol 271: 97-103, 2011.

34. Cedeno-Laurent F and Dimitroff CJ: Galectin-1 research in $\mathrm{T}$ cell immunity: past, present and future. Clin Immunol 142: 107-116, 2012

35. van der Leij J, van den Berg A, Harms G, et al: Strongly enhanced IL-10 production using stable galectin-1 homodimers. Mol Immunol 44: 506-513, 2007.
36. Stowell SR, Qian Y, Karmakar S, et al: Differential roles of galectin-1 and galectin-3 in regulating leukocyte viability and cytokine secretion, J Immunol 180: 3091-3102, 2008.

37. Mangan DF and Wahl SM: Differential regulation of human monocyte programmed cell death (apoptosis) by chemotactic factors and pro-inflammatory cytokines. J Immunol 147: 3408-3412, 1991

38. Liu F, Wu HY, Wesselschmidt R, Kornaga T and Link DC Impaired production and increased apoptosis of neutrophils in granulocyte colony-stimulating factor receptor-deficient mice. Immunity 5: 491-501, 1996.

39. Lieschke GJ, Grail D, Hodgson G, et al: Mice lacking granulocyte colony-stimulating factor have chronic neutropenia, granulocyte and macrophage progenitor cell deficiency, and impaired neutrophil mobilization. Blood 84: 1737-1746, 1994.

40. Shim W, Mehta A, Lim SY, et al: G-CSF for stem cell therapy in acute myocardial infarction: friend or foe? Cardiovasc Res 89: 20-30, 2011.

41. Schneider A, Krüger C, Steigleder T, et al: The hematopoietic factor $\mathrm{G}-\mathrm{CSF}$ is a neuronal ligand that counteracts programmed cell death and drives neurogenesis. J Clin Invest 115: 2083-2098, 2005

42. Schneider A, Kuhn HG and Schäbitz WR: A role for G-CSF (granulocyte-colony stimulating factor) in the central nervous system. Cell Cycle 4: 1753-1757, 2005.

43. Harada M, Qin Y, Takano H, et al: G-CSF prevents cardiac remodeling after myocardial infarction by activating the Jak-Stat pathway in cardiomyocytes. Nat Med 11: 305-311, 2005.

44. Fukada T, Hibi M, Yamanaka Y, et al: Two signals are necessary for cell proliferation induced by a cytokine receptor gp130: involvement of STAT3 in anti-apoptosis. Immunity 5: 449-460, 1996. 